\title{
Asentamiento de estadios tempranos de las centollas Lithodes santolla y Paralomis granulosa (Decapoda: Lithodidae) en colectores artificiales pasivos en el Canal Beagle, Argentina*
}

\author{
Federico Tapella ${ }^{1}$ \& Gustavo A. Lovrich ${ }^{1}$ \\ ${ }^{1}$ Consejo Nacional de Investigaciones Científicas y Técnicas (CONICET) \\ Centro Austral de Investigaciones Científicas (CADIC), Houssay 200, V9410CAB, Ushuaia, \\ Tierra del Fuego, Argentina
}

\begin{abstract}
RESUMEN. En el sector argentino del Canal Beagle, la pesquería de Lithodes santolla se encuentra colapsada y cerrada desde 1994, mientras que la del centollón Paralomis granulosa está estable, aunque por el crecimiento lento de la población no podría soportar alta presión de pesca. En este estudio se determinó la profundidad de reclutamiento de los estadios bentónicos tempranos y se evaluó la efectividad de colectores artificiales pasivos como oferta de refugio. En 2002 se fondearon colectores "SAC" en tres estratos de profundidad: 10-20, 20-40 y 40-90 m. En 2003, se colocaron colectores "Cajón" en dos estratos de profundidad: 10-20 y 20-40 m. En marzo de cada temporada se retiraron los colectores y se registró el número y tamaño de L. santolla y $P$. granulosa, y se determinó el taxón más abundante en cada colector. Los individuos del primer año de vida, i.e. $<5 \mathrm{~mm}$ largo de caparazón, reclutaron en los colectores. El reclutamiento de los primeros estadios bentónicos de L. santolla y P. granulosa ocurre sólo hasta los $40 \mathrm{~m}$ de profundidad. L. santolla seleccionaría ambientes dominados por poliquetos, y en menor medida por ofiuras y erizos, mientras que $P$. granulosa reclutaría en lugares dominados por almejas, anfípodos, ofiuras o erizos. La densidad de cangrejos en los colectores fue comparable con la encontrada en el grampón de Macrocystis pyrifera y en colectores similares para otras especies de crustáceos decápodos.
\end{abstract}

Palabras clave: cangrejos, colectores artificiales, subantártica, Macrocystis, arrecifes artificiales, Argentina.

\section{Recruitment of young-of-the-year lithodids Lithodes santolla and Paralomis granulosa in artificial passive collectors in the Beagle Channel, Argentina*}

\begin{abstract}
The L. santolla fishery in the Argentinean sector of the Beagle Channel collapsed and has been closed since 1994. Landings of $P$. granulosa in this sector are stable, but will not allow it to withstand heavy fishing pressure. In this study, we determined the recruitment depth of the early benthic stages of both lithodid species, and evaluated the effectiveness of passive artificial collectors as potential sources of refuge. In 2002, "SAC" collectors were deployed in three depth strata: 10-20, 20-40, and 40-90 m. In 2003, "Cajón" collectors were deployed in two depth strata: 10-20 and 20-40 m. The collectors were retrieved, respectively, in March 2003 and 2004, and the number and size of L. santolla and $P$. granulosa were recorded, along with the most abundant taxon in each collector. For both species, young-of-theyear individuals (i.e., $<5 \mathrm{~mm}$ carapace length) were found recruited in the collectors. Recruitment of the first benthic stages of $L$. santolla and P. granulosa occurs only to $40 \mathrm{~m}$ depth. $L$. santolla appears to select environments dominated by polychaetes and, to a lesser degree, by brittle stars and sea-urchins. $P$. granulosa seems to recruit in environments in which clams, amphipods, brittle stars, and sea-urchins dominate. Crab density in the collectors was comparable to that found for Macrocystis pyrifera and for other decapod species in similar collectors.
\end{abstract}

Key words: crabs, artificial collectors, Subantarctic, Macrocystis, artificial reefs, Argentine.

Autor corresponsal: Federico Tapella (tapella@tierradelfuego.org.ar)

* Trabajo presentado en el XXV Congreso de Ciencias del Mar de Chile y XI Congreso Latinoamericano de Ciencias del Mar (COLACMAR), realizados en Viña del Mar, entre el 16 y 20 de mayo de 2005. 


\section{INTRODUCCIÓN}

En aguas subantárticas del extremo austral de Sudamérica existen nueve especies de centollas (Thatje et al., 2005), de las cuales la mayoría tienen interés económico. Particularmente, en el canal Beagle se explotan comercialmente la centolla Lithodes santolla y el centollón o falsa centolla Paralomis granulo$s a$. La explotación de la centolla se desarrolló a partir de 1960 y alcanzó los máximos rendimientos (300 ton $\cdot a^{-1} 0^{-1}$ ) a mediados de la década de 1970. Desde entonces, los rendimientos declinaron al punto tal que promovieron la extracción del centollón o falsa centolla con menor valor comercial. Los desembarques de centollón en Ushuaia alcanzaron sus máximos (300 ton·año $\left.{ }^{-1}\right)$ durante la década del noventa (Lovrich, 1997). Los rendimientos alcanzan 3000

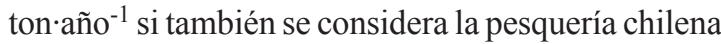
de la región de Magallanes, que se desarrolla desde Puerto Natales hasta el cabo de Hornos, incluyendo el canal Beagle y el estrecho de Magallanes. En la actualidad, la pesca está orientada principalmente al centollón y la centolla es sólo un subproducto de la pesca. Como la centolla es morfológicamente similar a los "king crabs" del Pacífico Norte, su demanda en los mercados ha sido siempre sostenida. Esto, sumado a la falta de controles pesqueros, causó el colapso de la pesquería mediante la extracción ilimitada de L. santolla, llevando los efectivos de la población a valores extremadamente bajos. En contraposición, el centollón es comparable a los "stone crabs" de calidad y precio más bajo en el mercado, y con una demanda discontinua que determina fluctuaciones en los desembarques. Esta circunstancia, sumada a la devolución de los animales menores a la talla legal, por no ser económicamente rentables, posiblemente evitó o retrasó el colapso de la población de centollón.

En los crustáceos decápodos, el reclutamiento a la fracción adulta y por ende el control del tamaño poblacional puede ocurrir durante el período larval (previo al asentamiento), el asentamiento y la selección del hábitat, o bien después de la metamorfosis a la fase juvenil habitualmente bentónica. En varias especies de interés comercial (e.g. cangrejos, langostas y langostinos), hay evidencias que indican que el reclutamiento a la fracción adulta y en consecuencia el rendimiento de la pesquería, está relacionado con la mortalidad de los primeros estadios bentónicos (Quinn \& Janseen, 1989; Wahle \& Steneck, 1991; Fernández et al., 1993; Iribarne et al., 1994; Eggleston \& Armstrong, 1995).
El uso de colectores artificiales para la captura de estadios larvales o juveniles de langosta Panulirus cygnus y "red king crab" Paralithodes camtschaticus, ha sido propuesto como una herramienta para predecir el rendimiento de la pesquería (Phillips, 1986; Forrest Blau \& Byersdorfer, 1994). En el canal Beagle, las larvas de $P$. granulosa son poco abundantes en muestras planctónicas, mientras que las de L. santolla estuvieron ausentes (Lovrich, 1999). Su comportamiento en experimentos de laboratorio está asociado al fondo del acuario (Anger et al., 2003; Lovrich et al., 2003; Calcagno et al., 2004) y por tanto se cree que las larvas de ambas especies son epibentónicas (Lovrich, 1999). Además, los primeros estadios bentónicos probablemente tengan un hábito críptico (Lovrich \& Vinuesa, 1995), y fueron encontrados, aunque en bajo número, solamente asociados a los grampones de fijación del alga Macrocystis pyrifera (M. Brusca, com. pers.). Por tal motivo, el objetivo de este estudio fue determinar la profundidad de reclutamiento y la efectividad en el asentamiento de estadios tempranos de P. granulosa y L. santolla en dos modelos de colectores artificiales pasivos.

\section{MATERIALES Y MÉTODOS}

Este estudio se efectuó en el archipiélago de las islas Bridges del canal Beagle ubicado en las proximidades a la ciudad de Ushuaia, Tierra del Fuego, Argentina (Fig. 1). En este sector del canal Beagle, la veda permanente a la pesca de $L$. santolla y $P$. granulosa se estableció en 1994 y existen evidencias de recuperación de la población de centollas.

Durante dos períodos de eclosión y probable asentamiento de estadios bentónicos de L. santolla y $P$. granulosa, agosto-marzo de 2002-2003 y 20032004, se fondearon colectores artificiales bentónicos que simulan la estructura tridimensional de los grampones de fijación de $M$. pyrifera. Los colectores fondeados fueron del tipo SAC ("Sausage-shaped Artificial Collector", modificado de Forrest Blau \& Byersdorfer, 1994) y Cajón. Los colectores SAC consistieron en una bolsa tubular de tejido multifilamento de $40 \times 60 \mathrm{~cm}$ de lado x $10 \mathrm{~cm}$ de alto y $1 \mathrm{~cm}$ de apertura de malla, rellena con $11 \mathrm{~m}^{2}$ del mismo tejido de multifilamento (Fig. 2a). Mientras que el colector del tipo Cajón consistió en un cajón plástico de $50 \times 30 \times 15 \mathrm{~cm}$, de pared perforada, relleno también con tejido de multifilamento y con $3 \mathrm{~kg}$ de lastre en su interior (Fig. 2b). El volumen de ambos colectores fue similar: 24 y $22,5 \mathrm{~L}$ para 


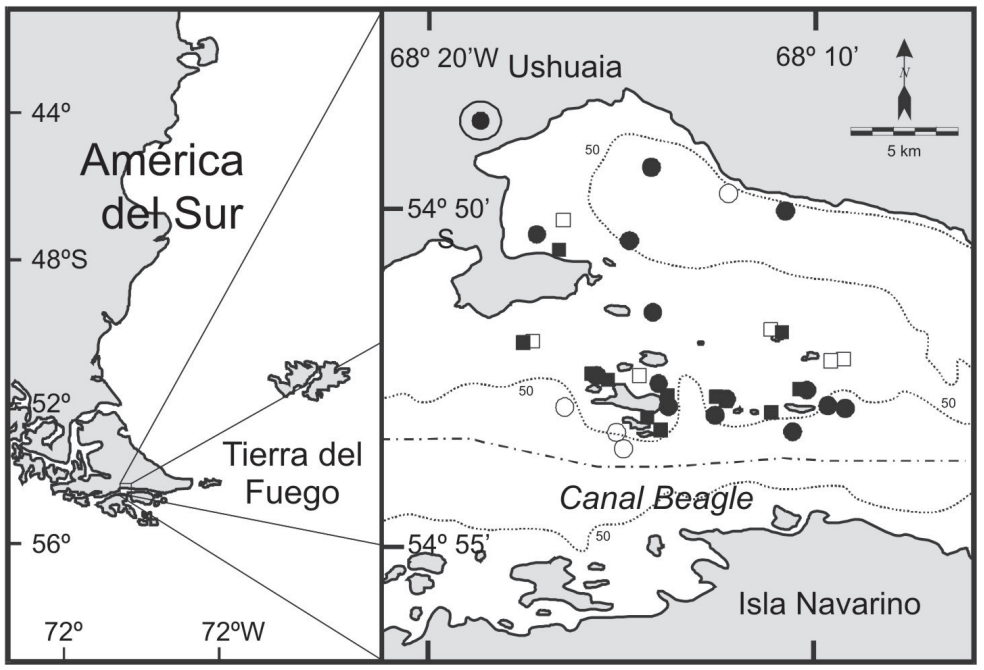

Figura 1. Ubicación geográfica de líneas de fondeo con colectores artificiales SAC (círculos) y Cajón (cuadrados) fondeados en el Canal Beagle para la captura de estadios tempranos de Lithodes santolla y Paralomis granulosa. Los círculos y cuadrados sin relleno representan líneas de fondo extraviadas de colectores SAC y Cajón, respectivamente.

Figure 1. Geographic location of longline gears deployed in the Beagle Channel with artificial collectors of two types: SAC (circles) and Cajón (squares) to capture young-of-the-year Lithodes santolla and Paralomis granulosa. Empty circles and squares indicate lost SAC and Cajón longlines, respectively.

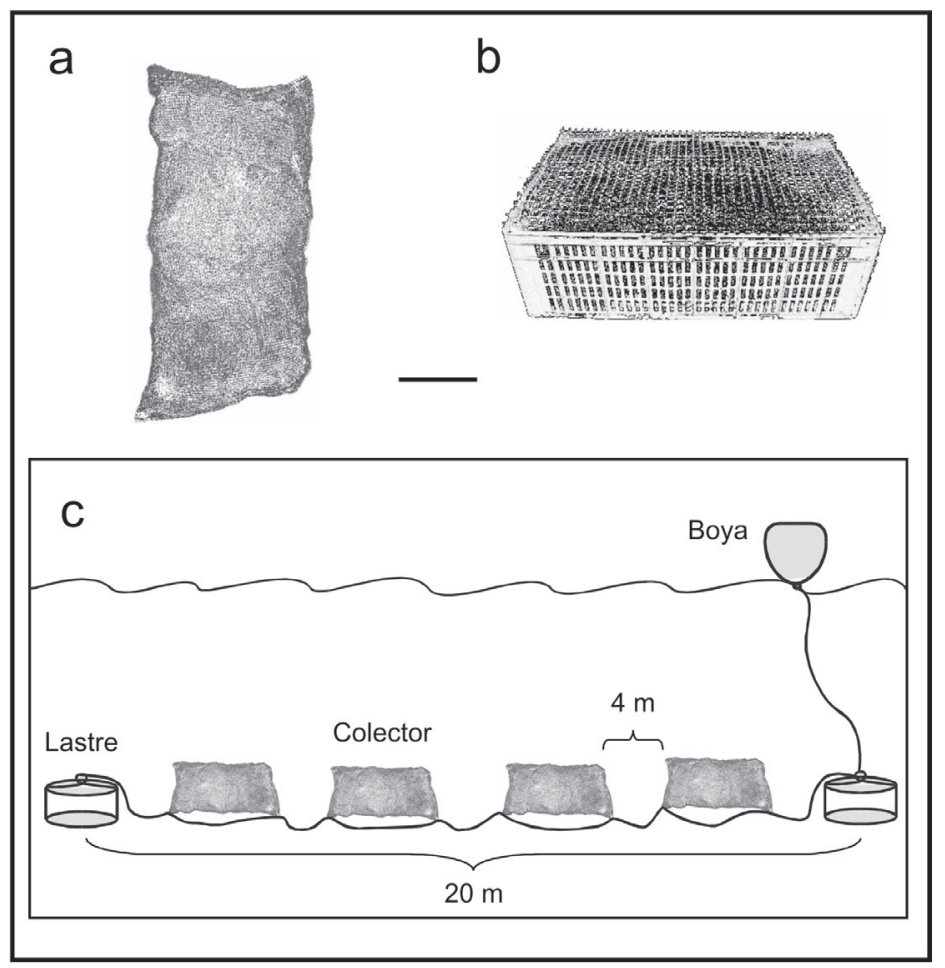

Figura 2. Aspecto general de colectores tipo a) SAC y b) Cajón, y c) esquema de la línea de fondeo utilizada para fondear ambos modelos de colectores en el Canal Beagle, Argentina. Barra $=25 \mathrm{~cm}$.

Figure 2. Collectors a) SAC and b) Cajón, and c) longline gear deployed in the Beagle Channel. $B a r=25 \mathrm{~cm}$. 
SAC y Cajón, respectivamente. La diferencia entre ambos modelos radica en su comportamiento bajo el agua. El colector SAC es más liviano y se mueve de lado a lado por acción de las corrientes, mientras que el tipo Cajón permanece fijo al fondo por el lastre. Los colectores de ambos modelos se dispusieron en líneas de fondeo, que consistieron en un cabo de 20 $\mathrm{m}$ con lastre en cada extremo, al que se amarraron cuatro colectores separados uno del otro por $4 \mathrm{~m}$. A ambos extremos del cabo se sujetó un lastre de 50 $\mathrm{kg}$ y a uno de ellos se amarró un cabo con una boya de flotación (Fig. 2c).

En agosto de 2002 se colocaron colectores SAC en tres estratos de profundidad: 10-20, 20-40 y 40-90 $\mathrm{m}$. En cada uno se fondearon 24 colectores dispuestos en seis líneas de fondeo. En agosto de 2003 se fondearon colectores Cajón en dos estratos: 10-20 y 20-40 m, colocando 36 colectores dispuestos en nueve líneas de fondeo por profundidad. En ambos años, las áreas seleccionadas para los estratos correspondientes a 10-20 y 20-40 m fueron al margen del bosque de $M$. pyrifera y la profundidad más próxima a este, respectivamente. En marzo de 2003 y 2004, se retiraron del agua los colectores SAC y Cajón, respectivamente, y fueron colocados en bolsas plásticas individuales y congelados a $-18^{\circ} \mathrm{C}$ hasta su procesamiento.

En el laboratorio se registró la cantidad de individuos de $L$. santolla y $P$. granulosa, y su tamaño (largo de caparazón, LC) se determinó bajo lupa estereoscópica con una precisión de $0,01 \mathrm{~mm}$. Además, se determinó cualitativamente el taxón dominante de la fauna acompañante de cada colector.

Para determinar si la abundancia de estadios tempranos de L. santolla y P. granulosa fue diferente entre los estratos de profundidad y tipo de colector, se realizaron análisis de varianza (ANOVA) de dos vías (Sokal \& Rohlf, 1995). Se planteó como hipótesis nula que la abundancia de ambas especies es independiente de la profundidad y tipo de colector utilizado. En ambos casos para comprobar normalidad y homogeneidad de varianza de cada grupo de datos, se utilizaron las pruebas de Kolmogorov-Smirnov y Bartlett, respectivamente (Sokal \& Rohlf, 1995). Para lograr estos supuestos los datos de abundancia (x) se transformaron según $\mathrm{y}=(\mathrm{x})^{-2}+(\mathrm{x}+1)^{-2}$.

\section{RESULTADOS}

En marzo de 2003, sobre un total de 72 colectores SAC colocados en 18 líneas de fondeo, se recupera- ron 19 colectores a $10-20 \mathrm{~m}, 19$ colectores a $20-40$ $\mathrm{m}$ y 15 colectores a $>40 \mathrm{~m}$ de profundidad, distribuidos en 14 líneas de fondeo (Fig. 1). En marzo de 2004, sobre la misma cantidad de colectores Cajón colocados en 18 líneas de fondeo, se recuperaron 24 colectores en cada estrato de profundidad, i.e., 10-20 m y 20-40 m, dispuestos en 12 líneas de fondeo (Fig. 1). El extravío de los colectores $\mathrm{SAC}$ fue porque se soltaron de la línea de fondeo o se perdieron las líneas completas. Los colectores Cajón sólo se perdieron por extravío de líneas de fondeo completas.

El asentamiento de los estadios tempranos de $P$. granulosa y $L$. santolla en colectores SAC varió con la profundidad. En el estrato de $>40 \mathrm{~m}$ de profundidad, ambos litódidos estuvieron ausentes, mientras que en los estratos de 10-20 y 20-40 m de profundidad, la abundancia promedio de ambas especies fue similar (Fig. 3, Tabla 1). Por tal motivo, durante el periodo de asentamiento 2003-2004 se fondearon colectores Cajón hasta $40 \mathrm{~m}$ de profundidad para contrastarlos con los colectores SAC. Al igual que ocurrió con los colectores SAC, en los colectores Cajón la abundancia promedio de $P$. granulosa y L. santolla fue similar entre los estratos de $10-20 \mathrm{y}$ 20-40 m de profundidad (Fig. 3, Tabla 1).

El reclutamiento de los estadios tempranos de estos litódidos fue diferente entre los colectores SAC y Cajón. La abundancia promedio de L santolla fue 3-4 veces mayor en los colectores Cajón que en los SAC (Fig. 3, Tabla 1), mientras que la abundancia de $P$. granulosa fue similar entre ambos colectores (Fig. 3, Tabla 1). Asimismo, la talla de los cangrejos recolectados fue diferente entre ambos colectores. En los colectores SAC, los cangrejos de $<5 \mathrm{~mm}$ LC de L. santolla y P. granulosa representaron el 100 y $80,6 \%$, respectivamente. Mientras que en los colectores Cajón, el mismo rango de tallas estuvo representada por el $75,9 \%$ y $51,6 \%$ de $L$. santolla y P. granulosa, respectivamente (Fig. 4).

Ambas especies se asentaron en forma diferencial en colectores colonizados por diferentes taxones. En ambos modelos de colectores se identificaron cinco taxones dominantes (Fig. 5). Los erizos fue el único taxón dominante en ambos modelos de colectores, mientras que almejas y anfípodos, y ofiuras y poliquetos fueron sólo dominantes en colectores SAC y Cajón, respectivamente (Fig. 5). Así, L. santolla ocurrió principalmente en colectores Cajón dominados por poliquetos y erizos, mientras que $P$. granulosa se encontró en colectores SAC y Cajón dominados por almejas, ofiuras y erizos (Fig. 5). 


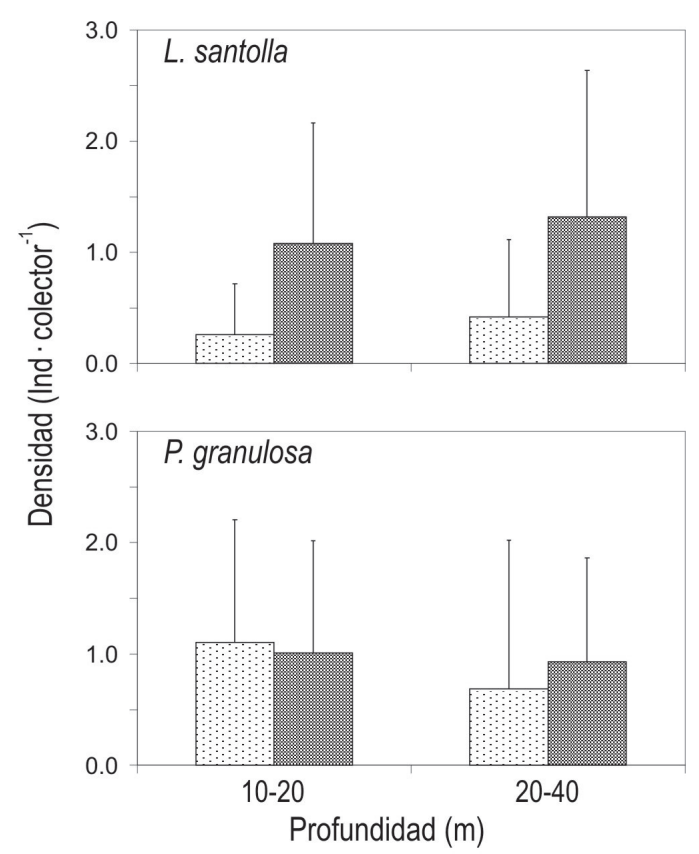

Figura 3. Abundancia promedio de estadios tempranos de Lithodes santolla y Paralomis granulosa en colectores artificiales tipo SAC y Cajón (columnas claras y oscuras, respectivamente) fondeados en dos estratos de profundidad en el Canal Beagle. El tamaño de la muestra fue de 19 y 24 colectores SAC y Cajón, respectivamente.

Figure 3. Average abundance of young-of-the-year Lithodes santolla and Paralomis granulosa settled in SAC and Cajón (light and dark columns, respectively) deployed at two depths in the Beagle Channel. Sample sizes were 19 and 24 for SAC and Cajón collectors, respectively.

\section{DISCUSIÓN}

Los resultados obtenidos constituyen las primeras observaciones sobre el reclutamiento de estadios tempranos de L. santolla y P. granulosa en colectores artificiales pasivos. Hasta el momento, los estudios realizados sobre estas especies fueron dirigidos al estudio de la biología básica para delinear normas de manejo y optimizar el rendimiento de la pesquería (Boschi et al., 1984; Wyngaard \& Iorio, 1996; Lovrich, 1997). Sin embargo, si bien la pesquería de ambas especies se desarrolla desde hace varias décadas, los estudios sobre el reclutamiento de los estadios larvales han sido escasos o nulos.

Los colectores artificiales capturaron principalmente los primeros estadios bentónicos, probablemente recién asentados. Las larvas de $P$. granulosa eclosionan entre junio y agosto (Lovrich \& Vinuesa, 1993) y las de L. santolla durante septiembre (Vinuesa, 1984). A la temperatura del canal Beagle durante agosto-noviembre, $c a .5$ a $7^{\circ} \mathrm{C}$ (Balestrini et al., 1998), el desarrollo larval hasta la metamorfosis al primer cangrejo de L. santolla y P. granulosa demanda 53 y 63 días, respectivamente (Anger et al., 2003, 2004). Posiblemente $P$. granulosa se asiente en el fondo entre agosto y octubre, y L. santolla a partir de noviembre. En el presente trabajo los estadios más frecuentes fueron $<4 \mathrm{~mm} \mathrm{LC}$, que corresponden a los cuatro primeros estadios, de aproximadamente 2,4; 2,9; 3,5 y 3,9 mm LC (Calcagno et al., 2005). En el laboratorio desde la eclosión hasta el estadio

Tabla 1. Análisis de varianza (ANOVA de dos vías) para poner a prueba las hipótesis nulas de igualdad en la abundancia de Lithodes santolla y Paralomis granulosa entre profundidad y tipo de colector. gl: grados de libertad, CM: cuadrados medios, F: estadístico $\mathrm{F}$, p: probabilidad que la hipótesis nula sea verdadera.

Table 1. Results of the two-ways ANOVA to test the effect of depth and type of artificial collector on the abundance of Lithodes santolla and Paralomis granulosa. gl: degrees of freedom, CM: mean squares, F: F-statistic, p: probability.

\begin{tabular}{lcccc}
\hline Fuente de variación & gl & CM & F & p \\
\hline Profundidad & 1 & 0,28 & 0,30 & 0,58 \\
$\quad$ Lithodes santolla & 1 & 3,36 & 2,83 & 0,10 \\
$\quad \begin{array}{l}\text { Paralomis granulosa } \\
\text { Colector }\end{array}$ & 1 & 12,12 & 13,15 & $<0,01$ \\
$\quad$ Lithodes santolla & 1 & 1,31 & 1,11 & 0,29 \\
$\quad$ Paralomis granulosa & & & & \\
Profundidad x colector & 1 & 0,03 & 0,03 & 0,86 \\
$\quad$ Lithodes santolla & 1 & 0,01 & 0,01 & 0,92 \\
$\quad$ Paralomis granulosa & & & & \\
Error & 82 & 0,92 & & \\
$\quad$ Lithodes santolla & 82 & 1,18 & & \\
$\quad$ Paralomis granulosa & & &
\end{tabular}




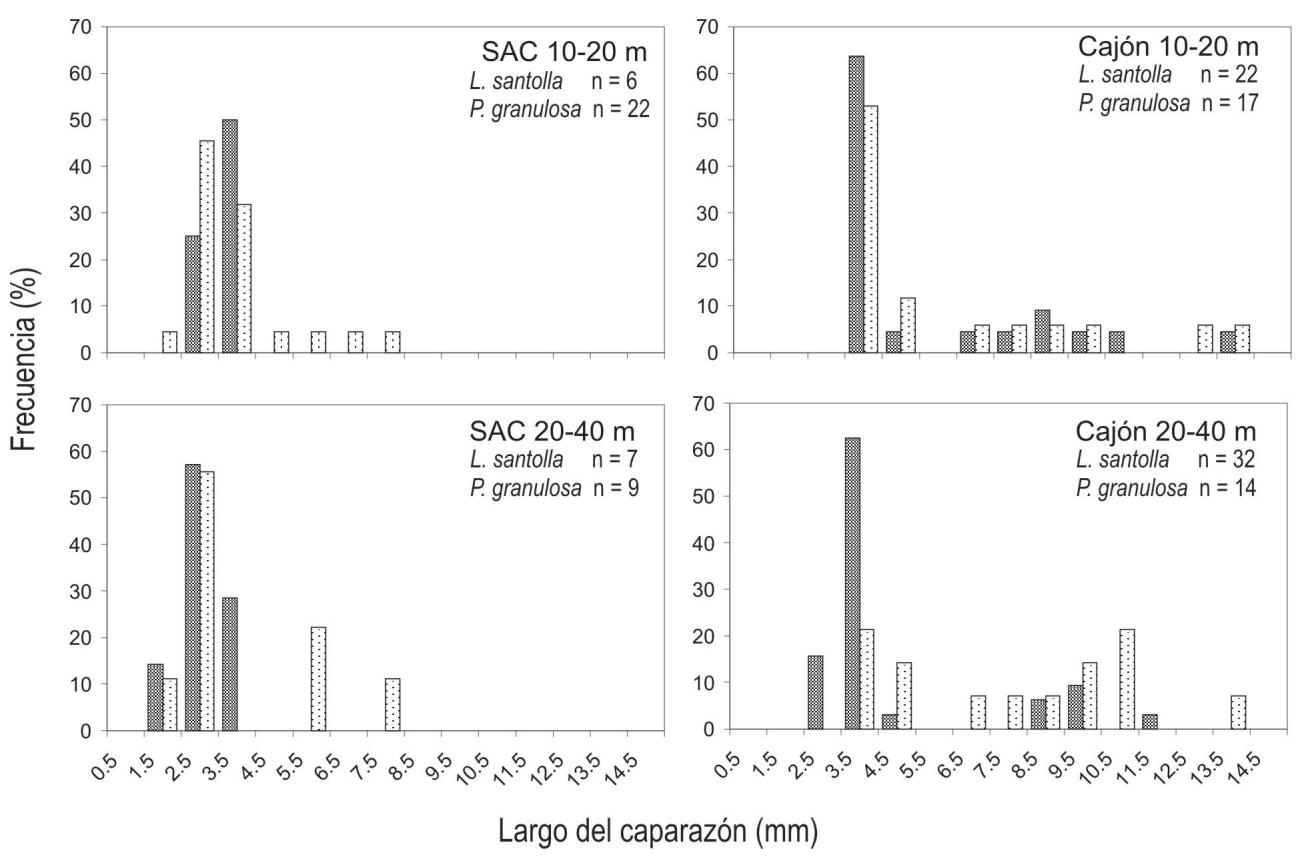

Figura 4. Distribuciones de frecuencia de tallas estadios tempranos de Lithodes santolla y Paralomis granulosa (columnas oscuras y claras, respectivamente) recolectados en colectores tipo SAC y Cajón fondeados en dos estratos de profundidad en el Canal Beagle. La cantidad de cangrejos recolectados de cada especie se expresa en cada gráfico.

Figure 4. Size frequency distributions of young-of-the-year Lithodes santolla and Paralomis granulosa (dark and light columns, respectively) captured with SAC and Cajón artificial collectors deployed at two depths in the Beagle Channel. Sample sizes for each species are shown in each graphic.

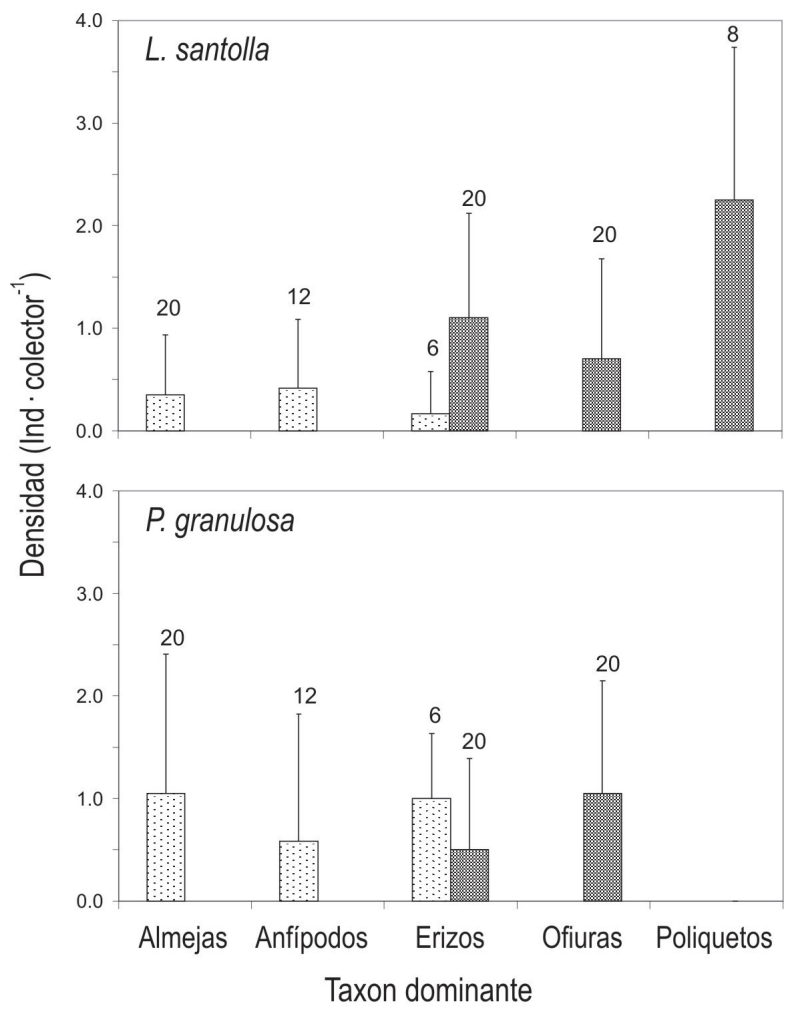

Figura 5. Abundancia promedio de estadios tempranos de Lithodes santolla y Paralomis granulosa en colectores SAC y Cajón (columnas claras y oscuras, respectivamente) dominados por diferentes taxones. Los números por encima de las barras de desvío indican la cantidad de colectores dominados por cada taxon.

Figure 5. Average abundance of young-of-the-year Lithodes santolla and Paralomis granulosa captured with SAC and Cajón (light and dark columns, respectively) collectors dominated by different taxa. Numbers above standard deviation lines represent the number of collectors dominated by each taxon. 
IV, P. granulosa requiere entre 266 y 163 días a 6 y $9^{\circ} \mathrm{C}$, y L. santolla entre 221 y 105 días, a idénticas temperaturas (Anger et al., 2003, 2004; Calcagno et al., 2005). Por tanto, los individuos que se asentaron en los colectores fueron mayoritariamente los que nacieron en invierno-primavera de la misma temporada en que se colocaron los colectores.

El uso de los colectores artificiales permitió determinar la distribución batimétrica de los estadios tempranos de L. santolla y P. granulosa. Los resultados obtenidos sugieren que el reclutamiento de estas especies ocurre sólo hasta $40 \mathrm{~m}$ y estaría relacionado con el rango de distribución batimétrica de Macrocystis pyrifera. Hasta el momento, los estadios tempranos de L. santolla y P. granulosa sólo han sido encontrados en colectores fondeados hasta 40 m (este estudio) y en los grampones de $M$. pyrifera (M. Brusca, com. pers.). En consecuencia, el rango de distribución batimétrico de los estadios tempranos de L. santolla y P. granulosa coincide con el rango máximo de distribución batimétrica de $M$. pyrifera. Esta alga se distribuye hasta los $\sim 40 \mathrm{~m}$ debido principalmente a la penetración de la luz (Kühnemann, 1970). El patrón de distribución batimétrico de $L$. santolla y $P$. granulosa es similar al observado para los estadios tempranos de Paralithodes camtschaticus del Pacífico norte, donde los primeros estadios bentónicos son más abundantes hasta los $50 \mathrm{~m}$ (Forrest Blau \& Byersdorfer, 1994).

Ambos modelos de colectores (SAC y Cajón) resultaron eficientes para la captura de estadios tempranos de L. santolla y P. granulosa, y comparable con los grampones de $M$. pyrifera. La estructura tridimensional y los espacios intersticiales logrados con el material de relleno (multifilamento) permitieron el asentamiento de cangrejos $<5 \mathrm{~mm}$ LC de $L$. santolla y $P$. granulosa. Al igual que ocurre en los grampones de M. pyrifera (M. Brusca, com. pers.), en ambos modelos de colectores, la mayoría (70-100\%) de los cangrejos recolectados fueron $<5 \mathrm{~mm}$ LC. Particularmente en los colectores Cajón, el porcentaje de cangrejos $>5 \mathrm{~mm}$ LC fue mayor que en los SAC. Probablemente, esta diferencia sea producto de la combinación entre el comportamiento de los colectores bajo el agua y el espacio intersticial del material de relleno. A diferencia de los colectores Cajón, los SAC se mueven bajo el agua. Este movimiento impediría que cangrejos $>5 \mathrm{~mm} \mathrm{LC}$, que no pueden acceder al centro del colector debido al tamaño de los espacios intersticiales, se mantengan en la periferia del colector SAC, y abandonen el colector.
La asociación entre la dominancia de un determinado taxón y la densidad de los litódidos podría indicar los hábitos alimenticios de los estadios tempranos. El desarrollo larval de ambas especies es endotrófico, durante el cual los individuos pierden peso (Calcagno et al., 2003; Lovrich et al., 2003) y seguramente los requerimientos energéticos luego de la muda son importantes. Los individuos de $P$. granulosa entre 10 y $20 \mathrm{~mm}$ LC se alimentan principalmente de organismos bentónicos sésiles, como briozoos e hidrozoos, pero también de moluscos y equinodermos (Comoglio \& Amin, 1999). Los estadios tempranos también pueden sobrevivir en el laboratorio con una dieta de nauplii de Artemia sp. (Calcagno et al., 2005), indicando que también son capaces de atrapar presas móviles. Los colectores donde se encontró $P$. granulosa estaban dominados por bivalvos (almejas) y equinodermos (erizos y ofiuras), que serían potenciales presas de esta especie, especialmente por tratarse también de individuos recientemente asentados. Además, $P$. granulosa también ocurrió en colectores dominados por anfípodos que podrían representar las potenciales presas móviles (Fig. 5). En L. santolla se conoce sólo la dieta natural de individuos $>40 \mathrm{~mm} \mathrm{LC}$, donde los equinodermos son una de las presas más frecuentes (Comoglio \& Amin, 1996). El asentamiento de estadios tempranos en colectores dominados por erizos y ofiuras recién asentados coincidiría con la elección de probables presas.

La densidad de individuos jóvenes de $L$. santolla y $P$. granulosa asentados en ambos modelos de colectores (SAC y Cajón), resultaron comparables con estudios similares en otras especies. En el "red king crab" P. camtschaticus el asentamiento en colectores dispuestos al azar fue de 1,1 ind colector $^{-1}$, llegando hasta 17 ind colector $^{-1}$ en los ubicados en lugares con mayor abundancia de adultos (Forrest Blau \& Byersdorfer, 1994; Loher \& Armstrong, 2000). Los mismos dispositivos fallaron en capturar los primeros estadios bentónicos de Chionoecetes bairdi, otra especie de interés comercial, aunque presentes en el área y asentados en otro tipo de colectores (Forrest Blau \& Byersdorfer, 1994). Jasus edwardsii se asienta en colectores similares a los de este estudio, a razón de 1,3 ind·colector ${ }^{-1}$, con mayores capturas en colectores con abertura de malla más pequeña (Mills \& Crear, 2004). El asentamiento de Homarus americanus depende estrechamente de la abundancia larval y ocurre en colectores rellenos con rodados a densidades de 0,8 a 2,3 ind $\cdot \mathrm{m}^{-2}$ (Incze et al., 1997). La abundancia promedio de $\sim 1,5$ ind $\cdot$ colector $^{-1}$ de $L$. 
santolla o $P$. granulosa indica que el diseño de los colectores es adecuado y eficiente para la captura de estas especies. Además, este valor es comparable con el encontrado en una estructura tridimensional natural, como el grampón de Macrocystis pyrifera (M. Brusca, com. pers.). El asentamiento en los colectores -y su éxito en la captura de individuos de una determinada especie dependerá de la provisión de larvas (Incze et al., 1997; Loher \& Armstrong, 2000), incluyendo fecundidad específica y mortalidad larval-, de la ventaja que ofrezcan los sustratos artificiales frente a los naturales en términos de depredación y competencia, y del tiempo de residencia en el colector.

\section{AGRADECIMIENTOS}

V. Mattenet, J. Neme, M. Diez, D. Pons, V. Murta, A. Ricci, M. Gutiérrez, A. Chizzini, C. Romero, P. Pérez Barros y M. Turus colaboraron en el trabajo de campo y/o de laboratorio. Se agradece las sugerencias sobre el texto y análisis estadístico a $\mathrm{C}$. Romero. Este estudio fue financiado por la Agencia Nacional de Promoción Científica y Tecnológica (PICT 01-10042) y la Internacional Foundation for Sciences (A-2507/2).

\section{REFERENCIAS}

Anger, K., G.A. Lovrich, S. Thatje \& J.A. Calcagno. 2004. Larval and early juvenile development of Lithodes santolla (Molina, 1782) (Decapoda: Anomura: Lithodidae) reared at different temperatures in the laboratory. J. Exp. Mar. Biol. Ecol., 306: 217-230.

Anger, K., S. Thatje, G.A. Lovrich \& J.A. Calcagno. 2003. Larval and early juvenile development of Paralomis granulosa reared at different temperatures: tolerance of cold and food limitation in a lithodid crab from high latitudes. Mar. Ecol. Prog. Ser., 253: 243-251.

Balestrini, C., G. Manzella \& G.A. Lovrich. 1998. Simulación de corrientes en el Canal Beagle y Bahía Ushuaia, mediante un modelo bidimensional. Servicio de Hidrografía Naval, 98: 1-58.

Boschi, E.E., D.A. Bertuche \& J.G. Wyngaard. 1984. Estudio biológico pesquero de la centolla (Lithodes antarcticus) del Canal Beagle, Tierra del Fuego, Argentina. Contr. Inst. Nac. Inv. y Des. Pesq., 441: 1-72.
Calcagno, J.A., K. Anger, G.A. Lovrich, S. Thatje \& A. Kaffenberger. 2004. Larval development of the subantarctic king crabs Lithodes santolla and Paralomis granulosa reared in the laboratory. Helgol. Mar. Res., 58: 11-14.

Calcagno, J.A., G.A. Lovrich, S. Thatje, U. Nettelmann \& K. Anger. 2005. Growth of young-ofthe-year lithodids, Lithodes santolla and Paralomis granulosa, reared at different temperatures. J. Sea Res., 54: 221-230.

Calcagno, J.A., S. Thatje, K. Anger, G.A. Lovrich \& A. Kaffenberger. 2003. Changes in biomass and chemical composition during lecithotrophic larval development of the southern stone crab Paralomis granulosa. Mar. Ecol. Prog. Ser., 257: 189-196.

Comoglio, L.I. \& O.A. Amin. 1996. Dieta natural de la centolla patagónica Lithodes santolla (Lithodidae) en el Canal Beagle, Tierra del Fuego, Argentina. Biol. Pesq., 25: 51-57.

Comoglio, L.I. \& O.A. Amin. 1999. Feeding habits of the false southern king crab Paralomis granulosa (Lithodidae) in the Beagle Channel, Tierra del Fuego, Argentina. Sci. Mar., 63 (Suppl. 1): 361-366.

Eggleston, D.B. \& D.A. Armstrong. 1995. Pre- and post-settlement determinants of estuarine dungeness crab recruitment. Ecol. Monogr., 65(2): 193-216.

Fernández, M., O. Iribarne \& D. Armstrong. 1993. Habitat selection by young-of-the-year dungeness crab Cancer magister Dana and predation risk in intertidal habitats. Mar. Ecol. Prog. Ser., 92: 171177.

Forrest Blau, S. \& S.C. Byersdorfer. 1994. Sausageshaped artificial collector developed in Alaska to study young-of-year red king crabs. Bull. Mar. Sci., 55(2-3): 878-886.

Incze, L.S., R.A. Wahle \& J.S. Cobb. 1997. Quantitative relationships between postlarval production and benthic recruitment in lobsters, Homarus americanus. Mar. Freshwater Res., 48: 729-743.

Iribarne, O., M. Fernández \& D. Armstrong. 1994. Does space competition regulate density of juvenile dungeness crab Cancer magister Dana in sheltered habitats? J. Exp. Mar. Biol. Ecol., 183: 259-271.

Kühnemann, O. 1970. Algunas consideraciones sobre los bosques de Macrocystis pyrifera. Physis, 29(79): 273-296. 
Loher, T. \& D.A. Armstrong. 2000. Effects of habitat complexity and relative larval supply on the establishment of early benthic phase red king crab (Paralithodes camtschaticus Tilesius, 1815) populations in Auke Bay, Alaska. J. Exp. Mar. Biol. Ecol., 245: 83-109.

Lovrich, G.A. 1997. La pesquería mixta de centollas Lithodes santolla y Paralomis granulosa (Anomura: Lithodidae) en Tierra del Fuego, Argentina. Invest. Mar., Valparaíso, 25: 41-57.

Lovrich, G.A. 1999. Seasonality of larvae of Brachyura and Anomura (Crustacea, Decapoda) in the Beagle Channel, Argentina. Sci. Mar., 63(Suppl. 1): 347-354.

Lovrich, G.A., S. Thatje, J.A. Calcagno, K. Anger \& A. Kaffenberger. 2003. Changes in biomass and chemical composition during lecithotrophic larval development of the southern king crab, Lithodes santolla (Molina). J. Exp. Mar. Biol. Ecol., 288: 65-79.

Lovrich, G.A. \& J.H. Vinuesa. 1993. Reproductive biology of the alse southern king crab (Paralomis granulosa, Lithodidae) in the Beagle Channel, Argentina. US. Fish. Bull., 91: 664-675.

Lovrich, G.A. \& J.H. Vinuesa. 1995. Growth of juvenile false southern king crab Paralomis granulosa (Anomura, Lithodidae) in the Beagle Channel, Argentina. Sci. Mar., 59(1): 87-94.

Mills, D. \& B. Crear. 2004. Developing a cost-effective puerulus collector for the southern rock lobster
(Jasus edwardsii) aquaculture industry. Aquacult. Eng., 31: 1-15.

Phillips, B.F. 1986. Prediction of commercial catchs of the western rock lobster Panulirus cygnus. Can. J. Fish. Aquat. Sci., 43: 2126-2130.

Quinn, J.P. \& J. Janssen. 1989. Crayfish comptetition in south-western Lake Michigan: a predator mediated bottleneck. J. Freshwater Ecol., 5: 75-85.

Sokal, R.R. \& F.J. Rohlf. 1995. Biometry, the principles and practice of statistics in biological research. W.H. Freeman, New York, 887 pp.

Thatje, S., K. Anger, J.A. Calcagno, G.A. Lovrich, H.O. Pörtner \& W.E. Arntz. 2005. Challenging the cold: crabs reconquer the Antarctic. Ecology, 86: 619-625.

Vinuesa, J.H. 1984. Sistema reproductor, ciclo y madurez gonadal de la centolla (Lithodes antarcticus) del Canal Beagle. Contr. Inst. Nac. Inv. Des. Pesq., 441: 75-95.

Wahle, R.A. \& R.S. Steneck. 1991. Recruitment habitats and nursery grounds of the American lobster Homarus americanus: a demographic bottleneck? Mar. Ecol. Prog. Ser., 69: 231-243.

Wyngaard, J.G. \& M.I. Iorio. 1996. Status of the southern king crab (Lithodes santolla) fishery of the Beagle Channel, Argentina. En: High latitude crabs: biology, management, and economics. Alaska Sea Grant College Program Report 96-02, University of Alaska Fairbanks, Alaska, pp. 25-39. 